\title{
A mini-microscope that moves with mice
}

Imaging methods for large-scale recordings of neural activity in rodents have improved significantly in recent years, but scientists still face significant hurdles, especially when trying to record activity at both high temporal and spatial resolutions from freelymoving animals. Many two-photon setups in awake-behaving mice can capture fast neural activity at the spatial resolution of dendritic spines $(\sim 1 \mu \mathrm{m})$, the sight of synaptic activity. But they are typically stand-alone benchtop rigs, requiring head-fixation of animals for imaging, lengthy training sessions, and a limited repertoire of expressed behaviors.

As an alternative to benchtop imaging platforms, miniaturized fiber-optic

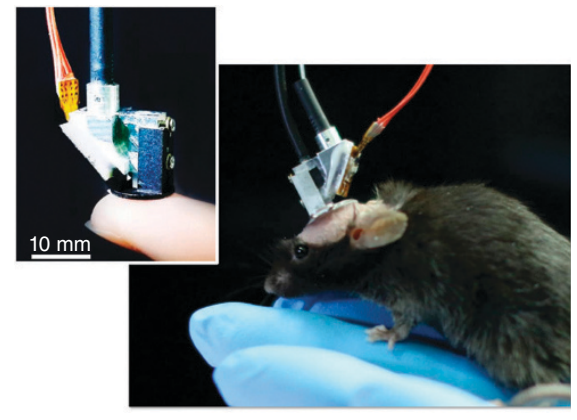

Picture of the miniature two-photon microscope alone and after implantation on a mouse. Adapted from Nat. Methods 14, 713-719 (2017).

imaging systems that attach directly to the animal's skull-and allow for free movement without head fixation - have been developed, but largely underperform in in vivo settings. In a new Nature Methods paper, Zong et al. describe the development of a new miniature two-photon microscope that weighs $\sim 2.15$ grams, can record fast activity from GCaMP labelled neuronal dendrites and spines, and provides steady images over hours in freely-moving mice (Nat. Methods 14, 713-719; 2017). Their mini-microscope significantly improves the quality of neural imaging in freely moving animals, and opens up opportunities to study neural circuits in greater detail during natural behaviors.

Dustin M. Graham 